\title{
DIRECTIONAL WAVELET TRANSFORMS AND FRAMES
}

\author{
Vladan Velisavljevic ${ }^{1}$, Pier Luigi Dragotti ${ }^{1}$, Martin Vetterli ${ }^{1,2}$ \\ ${ }^{1}$ Laboratoire de Communications Audiovisuelles (LCAV), École Polytechnique Fédéral de Lausanne \\ (EPFL), CH - 1015 Lausanne, Switzerland \\ ${ }^{2}$ Electrical Engineering and Computer Science Departmant, University of California at Berkeley, \\ Berkeley CA 94720
}

\begin{abstract}
The application of the wavelet transform in image processing is most frequently based on a separable transform. Lines and columns in an image are treated independently and the basis functions are simply products of corresponding onedimensional functions. Such a method keeps simplicity in design and computation. In this paper, a new two-dimensional approach is proposed, which retains the simplicity of separable processing, but allows more directionalities. The method can be applied in many areas like denoising, nonlinear approximation and compression. The results on nonlinear approximation and denoising show interesting gains compared to the standard two-dimensional analysis.
\end{abstract}

\section{INTRODUCTION}

Most wavelet transforms applied on images are separable transforms, that is, they treat lines and columns independently. The resulting basis functions are simply products of their one-dimensional counter parts.

The advantages of such an approach are conceptual simplicity, low computational complexity and inheritance of most properties from the one-dimensional case (e.g. regularity of the basis functions). The drawbacks are a partial treatment of the complexity inherent in two-dimensional images, which goes well beyond horizontal and vertical directions. Non-separable approaches [4], in particular using directional filter banks $[1,5]$, have been investigated, showing the potential of truly non-separable methods. Such methods come at a price in terms of design and computational complexity. Some separable approaches have been made in [9] but not on discrete space.

In the present paper, we wish to retain the simplicity of separable wavelet transforms while realizing some of the potential of non-separable schemes. We do this by introducing a directional wavelet transform that acts much like a standard separable transform, but allows more directionality. This is done by introducing "digital directions" that partition the discrete plane. Along these directions, it is then possible to apply orthogonal wavelet transforms or wavelet frame decompositions. Many useful properties are again inherited from the one-dimensional case (e.g. orthogonality). Techniques like denoising can be applied easily again by using more directions.

The outline of the paper is as follows. Section 2 defines digital directions and associated partitions of $\mathbb{Z}^{2}$. It also shows how directional elements in an image are treated by a separable directional transforms. Section 3 develops simple directional transforms and shows how to build a hierarchy of directional decompositions. Section 4 shows how to obtain orthogonal transforms and tight frames from these elementary directional transforms. Finally, Section 5 shows applications in non-linear approximation and denoising. We conclude in Section 6.

\section{DIGITAL DIRECTIONS}

A line in $\mathbb{R}^{2}$ is a simple object, but its equivalent in $\mathbb{Z}^{2}$ is a bit more complicated, as well known in raster graphics for example. A solution has been proposed in discrete tomography with the finite Radon transform [2] but involves wrap-around due to periodization. Here, we are interested in discrete approximations to real lines.

Then, we define a digital line of angle $\theta$ as a one-dimensional set of pixels approximately along a line of angle $\theta$. In addition, the digital line and its shifts along orthogonal direction have to tile $\mathbb{Z}^{2}$. While there are many solutions to this problem, a simple and tractable one is to use the analytical definition of a discrete line [3]. The line is determined by its slope and shift by the following equation:

$$
y[n]=\lfloor k x[n]\rfloor+\lfloor B\rfloor
$$

where $k=\tan (\theta)$ represents the slope and belongs to the range $0 \leq k \leq 1$, and $B$ represents the real-valued shift parameter. The definition of a discrete approximation of a line insures that each pixel belongs exactly to one line for a chosen slope. Lines with slopes out of the range may be obtained by symmetry, rotating and flipping vertically the space. This gives access to a wealth of directions in $\mathbb{Z}^{2}$. 
Building multi-resolution decompositions along multiple directions permits characterization and compression of phenomena other than just horizontal and vertical ones, as in the standard separable wavelet transform.

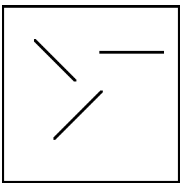

a)

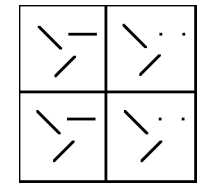

b)

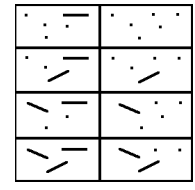

c)
Fig. 1. A simple object and its standard and directional transform; (a) Original image, (b) standard, horizontal-vertical transform, 1 step, (c) 3-direction transform, 1 step.

Figure 1 conceptually shows this. One step of a threedirection transform does isolate the different directions, as well as combinations thereof, and this in an intuitive way.

\section{SEPARABLE DIRECTIONAL TRANSFORM}

In this section, we concentrate on building some simple directional separable transforms. Among them, the simplest has four directions, namely $\left\{0^{0}, 45^{0}, 90^{0}, 135^{0}\right\}$.

A one-direction analysis leads to separation of an original image into two channels [8]. In the multi-directional case, one-directional analyses are applied direction by direction. Order is not important, since all combinations result in the same set of channels. For four-directional analysis, application of horizontal, vertical and both $45^{\circ}$ and $135^{\circ}$ (or $-45^{0}$ ) directional analysis gives 16 channels where each channel contains different combination of kept orientations.

Figure 2 shows an example of the multi-directional analysis. The original image contains four digital lines with different angles $\left(0^{0}, 45^{0}, 90^{0}\right.$ and $\left.135^{0}\right)$. The 4-direction transform (along $0^{0}, 45^{0}, 90^{0}$ and $135^{\circ}$ ) gives 16 sub-channels that keep different sets of angles each.

Iteration of such a transform is also possible. Some practical problems may appear in the downsampling of the transform sub-bands. The downsampling lattice may become extremely complex for a combination of the angles other than horizontal or vertical.

An example of the iteration of the 4-direction transform steps is shown in Figure $3 a$ ).

\section{DIRECTIONAL ORTHOGONAL TRANSFORMS AND FRAMES}

We show below that directional transforms, when built on top of one-dimensional orthogonal transforms or tight frames, again lead to orthogonal transforms or tight frames [7].

First, we need the following result. Assume a set of matrices corresponding to orthogonal transforms or tight

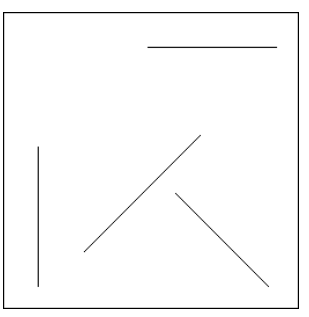

a)

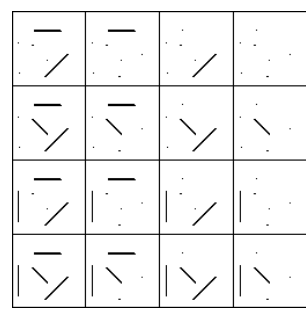

b)
Fig. 2. 4-direction transform of an image with 4 differently oriented lines. (a) Original image, (b) 16 transform channels.

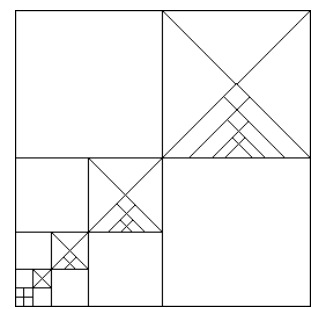

a)

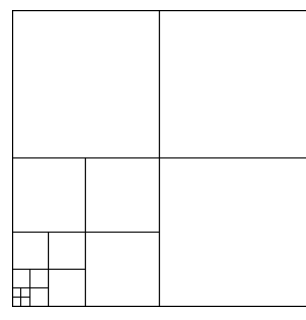

b)
Fig. 3. Two decomposition trees: (a) several steps of horizontal and vertical analysis followed by iterated steps of $\pm 45^{\circ}$-degree analysis applied on high-pass channels; $(b)$ several steps of horizontal and vertical analysis only.

frames, satisfying:

$$
F_{i}^{\mathrm{T}} \cdot F_{i}=\alpha_{i} \cdot I
$$

where $\alpha_{i}$ is some positive constant. Then, the frame $F$ defined as:

$$
F=\left[F_{1}^{\mathrm{T}} \ldots F_{N}^{\mathrm{T}}\right]^{\mathrm{T}}
$$

satisfies $F^{\mathrm{T}} \cdot F=\alpha \cdot I$, where $\alpha=\sum_{i=1}^{N} \alpha_{i}$.

So for example, taking an orthonormal wavelet transform on the rows of an image, and separately taking an orthonormal wavelet transform on the columns leads to a tight frame with $\alpha=2$.

For the purpose of proving the orthogonality of such directional transforms, consider an image as a set of real numbers defined on a finite lattice $\mathbb{R}^{N^{2}}$. We can divide this set into a series of subsets by taking directional lines at some angle as in (1). The division can be represented as $\mathbb{R}^{N^{2}} \rightarrow\left\{\mathbb{R}^{l_{1}}, \mathbb{R}^{l_{2}}, \ldots, \mathbb{R}^{l_{K}}\right\}$, where $l_{1}+l_{2}+\ldots+l_{k}=N^{2}$ and $K$ is the number of parallel lines. For example, for a transform along angle $\pm 45^{\circ}, K=2 N-1$.

If a one-dimensional unitary orthogonal transform is applied on the subsets it results in the same division of the space $\mathbb{R}^{N^{2}}$. Namely, for a set of transforms $\mathrm{U}=\left\{\mathrm{u}_{1}, \mathrm{u}_{2}, \ldots\right.$ $\mathrm{u}_{K}$ \}, the following holds:

$$
\mathrm{u}_{i}: \mathbb{R}^{l_{i}} \rightarrow \mathbb{R}^{l_{i}}, i=1,2, \ldots, K .
$$


The transform coefficients can be positioned at the same place in the lattice as the original pixels resulting in the same division of the space $\mathbb{R}^{N^{2}}$ as in the original image. Such a set of transforms produces a new set of coefficients that also belong to the original space $\mathbb{R}^{N^{2}}$. If a new set of unitary orthogonal transforms is applied along a different direction, the same equations hold again. Therefore, orthogonality holds for any particular direction and for any series of directions chosen.

Since orthogonality holds in any particular direction, a frame built from several directions is tight. The frame can be separated into two parts containing scaling and wavelet bases. Now, we can write:

$$
F_{i}^{\mathrm{T}} \cdot F_{i}=S_{i}^{\mathrm{T}} \cdot S_{i}+W_{i}^{\mathrm{T}} \cdot W_{i}=\alpha_{i} I,
$$

where $F_{i}=\left[S_{i}^{\mathrm{T}} W_{i}^{\mathrm{T}}\right]^{\mathrm{T}}$. For multiple directions, this separation principle allows us to write:

$F^{\mathrm{T}} \cdot F=\sum_{i=1}^{D} F_{i}^{\mathrm{T}} \cdot F_{i}=\sum_{i=1}^{D} S_{i}^{\mathrm{T}} \cdot S_{i}+W_{i}^{\mathrm{T}} \cdot W_{i}=\sum_{i=1}^{D} \alpha_{i}$

as it was proved above. Therefore, such a frame is tight regardless of the number of directions.

Since iteration is possible to be done on any sub-band channel, orthogonality and tightness hold for any step of iteration. Thus, they hold for the whole transform including all steps of iteration.

\section{APPLICATION IN NON-LINEAR APPROXIMATION AND DENOISING}

Application of the described method is possible in many areas of image processing where the standard wavelet transform is applied. Below, applications in non-linear approximation and denoising are presented.

\subsection{Non-linear approximation}

The number of significant coefficients produced by a horizontal and vertical transform depends on dominant orientation of objects in an image. If this direction is not matched with either the horizontal or vertical direction the number will increase. On the other hand, if some other directional transforms are applied on the channels, the number of significant coefficients related to those objects may decrease.

This property is exploited in non-linear approximation, where a fixed number of the largest coefficients is kept (the vectors that approximates the signal are adaptive and therefore the approximation is non-linear). A new decomposition tree is being compared to a standard one. The first is shown in Figure $3 a$ ) and the latter in Figure $3 b$ ). The quality of the reconstructed images in both cases are compared and the result of the comparison is expressed in terms of the PSNR factor. Figure $4 b$ ) shows the result of approximation of the image shown in Figure $4 a$ ), which is a synthetic randomly generated image that contains lines with different orientations. These results indicate that the new method can be attractive in non-linear approximation.

\subsection{Denoising}

The standard denoising process is usually done by thresholding of coefficients obtained by a transform along horizontal and vertical direction. Some difficulties may appear since visibility of objects in an image depends on their orientations.

Also, one-dimensional denoising of an image is not able to catch most of the two-dimensional interdependencies present in images. This is the reason why independent denoising in only two directions (line-by-line, or column-bycolumn) of an image does not necessarily give the best result.

However, such an approach has the main advantage of simplicity. In trying to retain this simplicity, but still making a method that better exploits the two-dimensional characteristics of an image, we introduce multiple directional denoising. An image is processed by taking sets of pixels in different orientations and denoising them by hard thresholding [6] of wavelet coefficients. The result is compared to the result of a standard method where horizontal and vertical wavelet decomposition was done in several levels and the coefficients were hard thresholded as well.

Figure 5 shows an example of denoising of the image 'Cameraman'. The original image is affected by additive Gaussian noise and both methods were applied. The dependence of the performance of the denoising on the number of directions in the analysis is shown in the Figure $6 a$ ). The comparison between the new and the standard method is shown in the Figure $6 b$ ). The new method strongly outperforms the standard one.

\section{CONCLUSION}

The standard method of the wavelet decomposition of images using separable bases involves only two directions of analysis. Our new method goes beyond this limitation and calculates separable wavelet transform along sets of different directions. Such an approach takes into account twodimensional interdependencies among image pixels better than the standard method. Simplicity and low computational complexity are still maintained. Application of the new method is possible in various areas of image processing. Outperforming results are shown in non-linear approximation and denoising. 


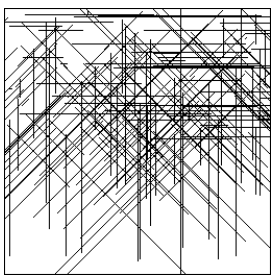

a) b)

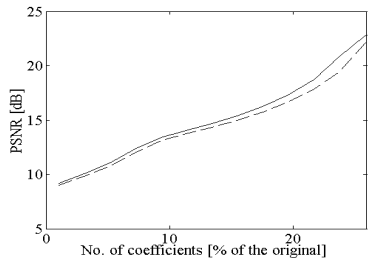

Fig. 4. Result of non-linear approximation. (a) Original image, (b) Results: full line shows the dependence of PSNR of the reconstructed image on the number of coefficients kept for the new method. The dotted line shows the same dependence for the standard method.

\section{ACKNOWLEDGMENTS}

The third author would like to thank Prof. Minh Do for fruitful interactions on directional image analysis.

\section{REFERENCES}

[1] R. H. Bamberger and M. J. T. Smith. A filter bank for the directional decomposition of images: Theory and design. IEEE Signal Proc., pages 882-893, April 1992.

[2] E. D. Bolker. The finite Radon transform. In S. Helgason R. L. Bryant, V. Guillemin and R. O. Wells Jr., editors, Integral Geometry (Contemporary Mathematics, Vol. 63), pages 2750. 1987.

[3] J. E. Bresenham. Algorithm for computer control of a digital plotter. IBM Systems Journal, 4(1):25-30, 1965.

[4] E. J. Candès and D. L. Donoho. Curvelets, multiresolution representation, and scaling laws. In A. Aldroubi, A. F. Laine, and M. A. Unser, editors, SPIE Wavelet Applications in Signal and Image Processing VIII, volume 4119, 2000.

[5] M. N. Do. Directional Multiresolution Image Representations. PhD thesis, Audio-Visual Laboratory, EPFL, October 2001.

[6] D. L. Donoho and I. M. Johnstone. Ideal spatial adaption via wavelet shrinkage. Biometrika, pages 425-455, December 1994.

[7] V. K. Goyal, J. Kovačević, and J. A. Kelner. Quantized frame expansions with erasures. Applied \& Computational Harmonic Analysis, 10(3):203-233, May 2001.

[8] M. Vetterli and J. Kovačević. Wavelets and Subband Coding. Prentice Hall PTR, New Jersey, 1995.

[9] R. A. Zuidwijk. Directional and time-scale wavelet analysis. SIAM Journal on Mathematical Analysis, 31(2):416-430, 2000.

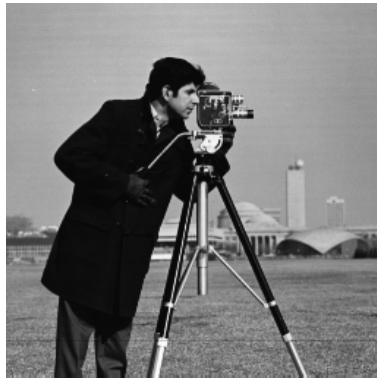

a)

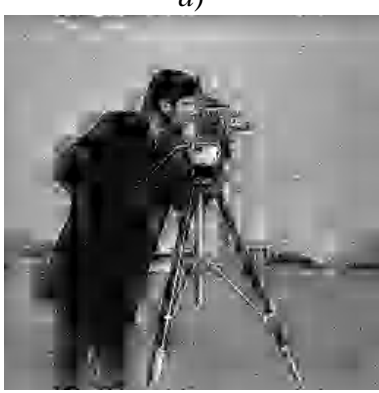

c)

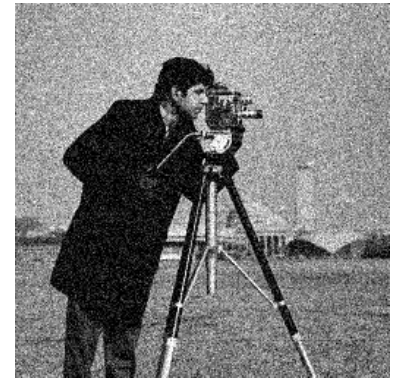

b)

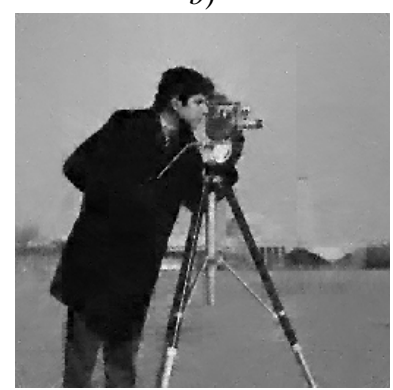

d)
Fig. 5. The image 'Cameraman'. (a) Original image, (b) noised version $(P S N R=19 d B),(c)$ denoised by the standard method $(P S N R=20.54 \mathrm{~dB}),(d)$ denoised by the new method $(P S N R=$ $25.04 \mathrm{~dB})$

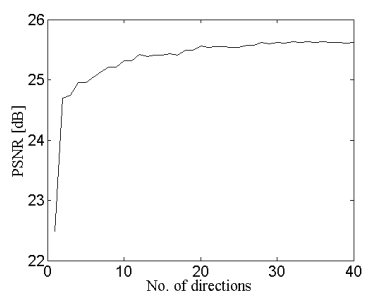

a)

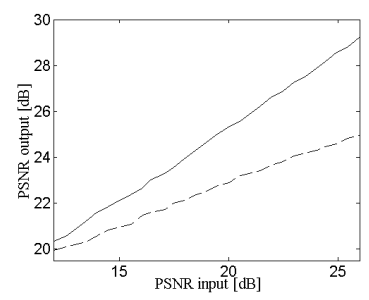

b)
Fig. 6. Result of denoising of the image 'Cameraman'. (a) The dependence of $P S N R$ of the denoised image on the number of the directions involved in the analysis, $(b)$ The full line shows the dependence of $P S N R$ of the denoised image on the input $P S N R$ for the new method. The dotted line shows the same dependence for the standard method. 